\title{
PQLI Key Topics - Criticality, Design Space, and Control Strategy
}

\author{
Thomas Garcia • Graham Cook • Roger Nosal
}

Published online: 19 May 2008

(C) International Society for Pharmaceutical Engineering 2008

\begin{abstract}
This paper gives an overview of progress made by the ISPE PQLI initiative - a global industry-led initiative aimed at facilitating the implementation of ICH Q8, Q9, and ultimately Q10 guidance. Through this initiative ISPE is spearheading the effort to help industry begin to define areas where they will be able to provide the technical framework for the implementation of key elements of Quality by Design (QbD) - a systematic approach to development that begins with predefined objectives and emphasizes product and process understanding based on sound science and quality risk management. Three topic areas, Design Space, Criticality, and Control Strategy were selected for specific focus and discussion, and this paper gives an overview of progress in these three areas.
\end{abstract}

Keywords PQLI · Design space · Criticality · Control strategy $\cdot$ Critical quality attribute $\cdot$ Critical process parameter $\cdot$ Knowledge space $\cdot$ PAT $\cdot$ Pharmaceutical target product profile · Product and process knowledge - Quality by design - Quality risk management

\footnotetext{
T. Garcia $\cdot$ R. Nosal

Pfizer Inc.,

Eastern Point Rd., Groton,

CT 06340, USA

T. Garcia

e-mail: thomas.p.garcia@pfizer.com

R. Nosal

e-mail: roger.nosal@pfizer.com

G. Cook

Wyeth Pharmaceuticals,

New Lane, Havant,

PO9 2NG, UK

e-mail: cookg1@wyeth.com
}

Contributing Team Members Bruce Davis - AstraZeneca, Line Lundsberg - NNE Pharmaplan, Stephen Tyler - Abbott, James Spavins - Pfizer, PQLI Criticality Task Team, PQLI Design Space Task Team, PQLI Control Strategy Task Team

\section{Introduction}

The International Society for Pharmaceutical Engineering (ISPE) launched the Product Quality Lifecycle Implementation (PQLI) initiative in June 2007 in US [1] and a follow-up workshop was held in Europe in September [2]. The intention of PQLI is to work with industry and regulatory agencies worldwide to facilitate a common understanding of Quality-by-Design (QbD), and introduce pragmatic and practical means for the implementation of ICH guidance's, based on sound scientific, engineering and business principles. The emphasis will initially be on providing 'how to' implementation guidance on ICH Q8, Q8 (R), Q9 and Q10 [3-6]. It will embrace science and engineering, cover small and large molecules, and address both drug substance and drug product.

PQLI outputs are not regulatory documents. They try to capture best practice and draw their sources from open intra-industry and regulatory discussions aimed at practical solutions to ensure product quality, and as such this paper represents a work-in-progress. It is important to note that PQLI is not in place of or in competition with the ICH process and its goals. Rather, it is a process committed to helping out with the implementation of ICH guidance.

PQLI issued a summary update report September 14, 2007 [7], which coincided with the European ISPE PQLI Conference in Berlin, Germany. Additional discussions on the task team's progress with regulators from the US, Europe and Japan occurred at the 2007 ISPE Annual Meeting [8, 9]. During this conference, opportunities to broaden the program to biotechnology products were also explored. A further major workshop was held during the ISPE Congress in Copenhagen [16].

This document provides an overview of the progress thus far for the Criticality, Design Space, and Control Strategy task teams. It is part of a suite of documents to be produced by ISPE. 
This paper gives an overview of the progress on the topics of Criticality, Design Space, and Control Strategy, and how the Design Space and Control Strategy may be presented.

This should be regarded as work in progress and comment and input from industry, regulators, and other stakeholders is welcomed.

\section{Background}

In discussing the Desired State ${ }^{1}$ Janet Woodcock $[10,17]$ described Quality by Design (QbD) as encompassing the development of scientific understanding of critical process and product attributes, designing controls and testing based on the limits of scientific understanding at development stage, and utilizing the knowledge gained over the product's lifecycle to operate in an environment of continuous improvement. Recently, the International Conference on Harmonization (ICH) has defined QbD in ICH $\mathrm{Q} 8 \mathrm{R}$ as "a systematic approach to pharmaceutical development that begins with predefined objectives and emphasizes product and process understanding based on sound science and quality risk management."

Some elements of QbD have been used for many years. For example, the use of statistically designed experiments (DOE) dates back to the 1920's as factorial designs were applied in agricultural science, and the 1950's when they were more widely used for industrial applications. FMEA, a commonly used risk assessment tool, was developed by the United States Military to assess equipment and system failures [11]. In the 1990's, software was developed that combined risk assessment and DOE techniques. The spotlight on these techniques has intensified in the pharmaceutical industry, in particular with the United States Food and Drug Administration's (FDA) issuance of their report "Pharmaceutical CGMPs for the 21st Century: A Risk-Based Approach; A Science and Risk-Based Approach to Product Quality Regulation Incorporating an Integrated Quality Systems Approach" [12]. This report launched a strategic change towards the presentation of more scientific knowledge in submissions, thereby laying the groundwork for QbD. Shortly afterwards, FDA issued the guidance document, "PAT — A Framework for Innovative Pharmaceutical Development, Manufacturing, and Quality Assurance" [13]. Although the focus was more geared towards process analytical technology (PAT), this guidance document discussed many principles of $\mathrm{QbD}$. Subsequent papers followed, such as the EMEA PAT

\footnotetext{
${ }^{1}$ The Desired state has been described as one in which Manufacturers have extensive knowledge about critical product and process parameters and quality attributes, and strive for continuous improvement. The regulator's role is initial verification, subsequent auditing, leading to no manufacturing supplements.
}

Team's reflection paper which highlighted how PAT could result in real-time release and play a pivotal role in Quality by Design [14]. A literature search would produce numerous more examples of papers on topics related to $\mathrm{QbD}$ and design space.

In 2005, ICH Q8 was issued which focused on the content of Section 3.2.P.2 of the Common Technical Document (CTD) and introduced the concept of design space $^{2}$. An important step in defining the design space involves the differentiation between those product attributes and process parameters that are critical from those that are not. One common approach to achieve such decisions is the use of risk assessment. ICH Q9 was issued, which discusses potential approaches and tools that could be used to perform risk assessments, as well as the management of identified risks. The final document of the tripartite is $\mathrm{ICH}$ $\mathrm{Q} 10$, which addresses the quality management systems of pharmaceutical manufacturers. This guidance outlines expectations for the Pharmaceutical Quality System $(\mathrm{PQS})^{3}$, and how they can be applied in the management of the design space, risk assessment, and to ensure quality standards are met over the lifecycle of the product. ICH Q8 (R), currently at Step 2, describes the principles of QbD and provides further clarification of key concepts outlined in ICH Q8. This annex is intended to show how concepts and tools could be put into practice by the applicant for all dosage forms. At the time of writing, both ICH Q10 and Q8 (R) are still subject to revision.

The use of $\mathrm{QbD}$ principles during product development provides opportunities to facilitate innovation and continual improvement throughout the product lifecycle, compared to traditional approaches. QbD principles increase process knowledge and product understanding, often through the application of new technologies such as PAT or modeling. Prior knowledge and experience may be leveraged when performing risk assessments and planning statistically designed experiments. Information from these experiments can improve the quality of the product and enhances the ability of regulatory reviewers to make an accurate assessment of the drug substance and drug product development and manufacturing sections of the Common Technical Document (CTD), leading to first cycle approval.

\footnotetext{
${ }^{2}$ ICH Q8 defines design space as "the multidimensional combination and interaction of input variables (e.g., material attributes) and process parameters that have been demonstrated to provide assurance of quality. Working within the design space is not considered as a change. Movement out of the design space is considered to be a change and would normally initiate a regulatory post approval change process. Design space is proposed by the applicant and is subject to regulatory assessment and approval."

${ }^{3}$ ICH Q10 defines a Pharmaceutical Quality System as a "management system to direct and control a pharmaceutical company with regard to quality."
} 
The increased process knowledge and product understanding resulting from QbD can increase the efficiency of manufacturing processes, reduce product recalls and compliance actions, resulting in cost savings for pharmaceutical companies. By reducing uncertainty and risk, $\mathrm{QbD}$ can allow industry and regulators to focus their resources in the most critical areas. Because much more process understanding has been demonstrated and expressed in the dossier, QbD filings also can help facilitate CMC reviews and GMP inspections by the regulators and decrease the number of post-approval regulatory submissions required to make process changes. QbD can also facilitate the use of innovative technologies and promote the use of new approaches to perform process validation, such as continuous quality verification.

\section{Progress on PQLI Topic Areas}

Three key topic areas were selected for discussion and investigation:

- Critical vs. Non-Critical

- Design Space

- Control Strategy (traditional vs. enhanced approaches based on QbD concepts)

A separate Task Team addressed each of the topics. Each Team considered both the active pharmaceutical ingredient (API) and drug product.

Criticality determines what quality attributes and process parameters are defined in the Design Space. The Design Space defines the relationship between Critical Quality Attributes (CQAs) and Critical Process Parameters (CPPs), and identifies acceptable operating ranges for CPPs. It is the region where acceptable product can be produced. The normal operating range is a subset of the Design Space where routine manufacture is typically performed on a daily basis. Finally, the Control Strategy ensures that operation of the process is maintained within the Design Space. It is intended to prevent operating in regions of limited process knowledge or that are known to cause product failure.

Figure 1 shows how these three elements are connected and interact with each other. The Knowledge Space is a summary of all process knowledge obtained during product development. It includes information about critical and non-critical attributes and process parameters. This encompasses the Design Space and normal operating ranges, as well as areas where it is known that unacceptable product is produced. The Knowledge Space only contains information regarding regions that have been investigated, and beyond its boundaries is considered to be unexplored space.

Detailed discussion is presented for each topic in the following sections.

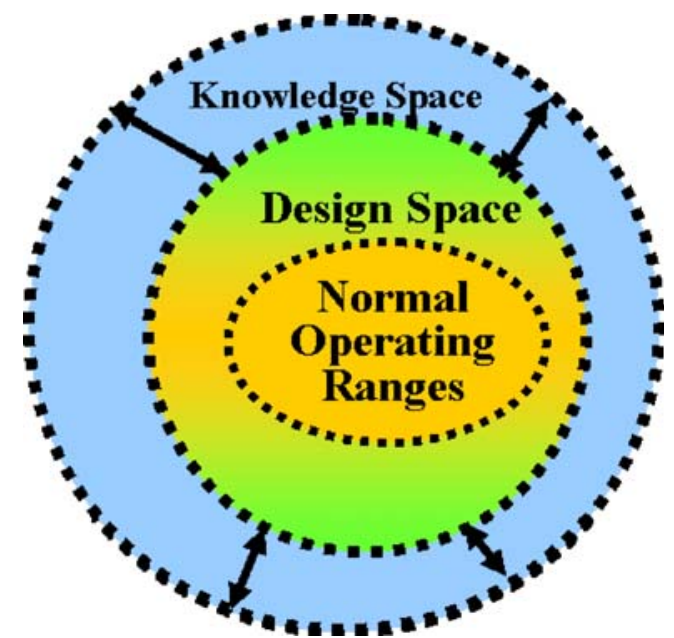

Fig. 1 Linkage between knowledge space, design space, and normal operating ranges

\section{Criticality}

The concept of criticality can be used to describe any feature or material attribute, property or characteristic of a drug substance, component, raw material, drug product or device, or any process attribute, parameter, condition or factor in the manufacture of a drug product. The assignment of attributes or parameters as critical or non-critical is an important outcome of the development process that provides the foundation for deciding what is or isn't included in the Design Space. The Criticality Task Team concluded that establishing criticality is a process, rather than a simple definition. Underlying the process is the concept that the primary assessment and designation of criticality should be made relative to the impact that quality attributes or process parameters have on the safety, efficacy and quality ${ }^{4}$ of the product. In addition, the team looked for consistency with current accepted definitions and alignment with ICH guidance.

Figure 2 shows a proposed decision tree for establishing criticality. The diagram is divided into foundational and developmental areas. The foundational portion of the decision tree determines whether an enhanced or minimal approach to pharmaceutical development will be pursued. A preliminary assessment of "business" attributes is performed at this point. The developmental area of the diagram distinguishes categories of criticality, first the degree of severity and then levels of risk based on probability and detectability.

The process applies a series of filtering questions to determine if an attribute or process parameter impacts the safety, efficacy, or quality of the product. It relies on $\mathrm{QbD}$

\footnotetext{
${ }^{4}$ Quality is defined as the suitability of either a drug substance or drug product for its intended use. This term includes such attributes as the identity, strength, and purity [15].
} 
Fig. 2 Decision tree to define levels of criticality
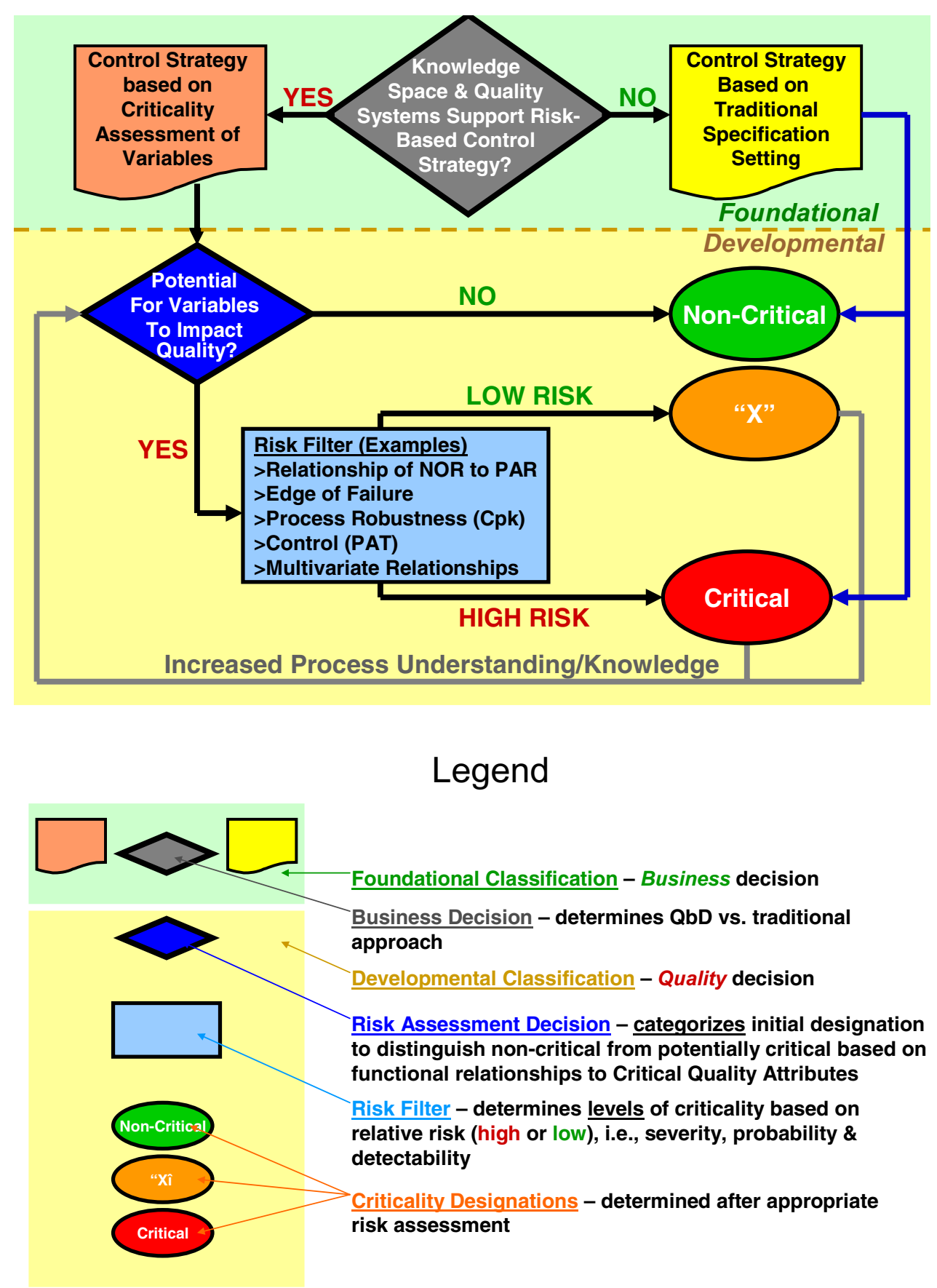

elements including risk assessment, the establishment of a Design Space, and the development of a Control Strategy. As the questions are answered, an attribute or parameter is taken down a specific path that categorizes its degree of criticality. With increased process knowledge and understanding, quality attributes and process parameters can undergo multiple iterations to reclassify their categorization, as necessary.

Risk assessments should consider cause and effect relationships, relative to probability, severity, detectability, and sensitivity. Probability is the likelihood of harm occurring, while severity is the measure of the possible consequence. Detectability refers to the ability to discover or determine the existence, presence, or fact of a hazard, and sensitivity is the attenuation of interactions between multivariate dimensions. ${ }^{5}$ Using descriptive adjectives to

\footnotetext{
${ }^{5}$ Univariate analysis is an approach that may contribute to the creation of a design space. However, its application in conjunction with multivariate approaches may warrant additional studies to address interactions.
} 
Fig. 3 Proposed model for control strategy
PATIENT

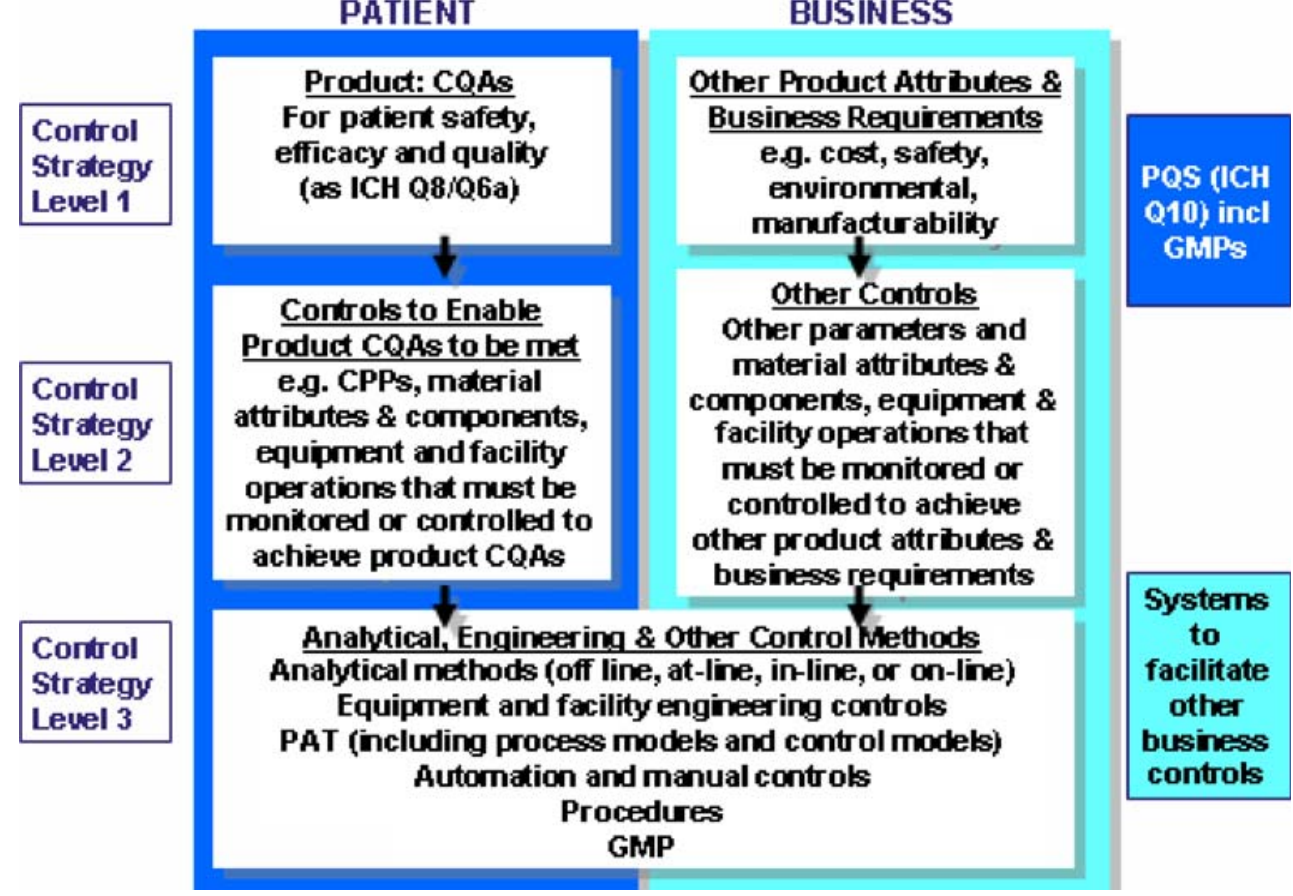

define criticality with regard to these four elements clarifies the context associated with the risk.

Several levels of criticality may be used to describe multiple levels of risk. As the boundaries for a quality attribute or parameter approach edges of failure, the level of criticality increases with the level of risk. Following the risk assessment, some companies may choose to introduce additional optional terms such as 'key' or 'important'. Figure 2 therefore includes an additional category (" $\mathrm{X}$ ") between the critical and non-critical classifications, to reflect this option.

The purpose of this intermediate category is to address those attributes or parameters that may impact the safety, efficacy or quality of the product, but to a lesser degree than what is observed for other CQAs or CPPs. Attributes and process parameters in this intermediate category still warrants some attention, and their importance should not be overlooked; which could occur if they were categorized as being non-critical.

Note that a Control Strategy or test does not make a CQA or CPP non-critical; but rather makes it controlled. As additional process information is obtained over the lifecycle of the product, it is possible that the criticality of some attributes or parameters may change. In such instances, changes in designation from one level of criticality to another must be demonstrated by data. If the change requires a change to the Control Strategy, then some measure of notification to regulatory authorities is required. Changes that do not result in a change to the Control Strategy may not require such notification.
Further details may be found in the paper by the PQLI Criticality Task Team: Criticality in PQLI [18] in this Volume of the Journal of Pharmaceutical Innovation.

\section{Design Space}

The development and refinement of the Design Space begins at product conceptualization and continues to evolve throughout the lifecycle of the product. At the time of filing a submission, the Design Space can be considered to be a snap-shot in time representative of the current process knowledge. It continues to evolve as additional knowledge and information is generated during the commercialization of the product, which may lead to post-approval changes. Movement out of the Design Space is considered to be a change and would normally initiate a regulatory post approval change process. As such, the Design Space will require management under a company's Pharmaceutical Quality System.

The creation of a Design Space begins with the definition of the Pharmaceutical Target Product Profile (PTPP) ${ }^{6}$, which identifies the desired performance characteristics of the product. Prior knowledge and a preliminary risk assessment can be used to identify experiments to be

\footnotetext{
${ }^{6}$ ICH Q8R defines a PTPP as being "a prospective and dynamic summary of the quality characteristics of a drug product that ideally will be achieved to ensure that the desired quality, and hence the safety and efficacy, of a drug product is realised."
} 
Fig. 4 Summary of drug product process knowledge for an oral solid dosage form

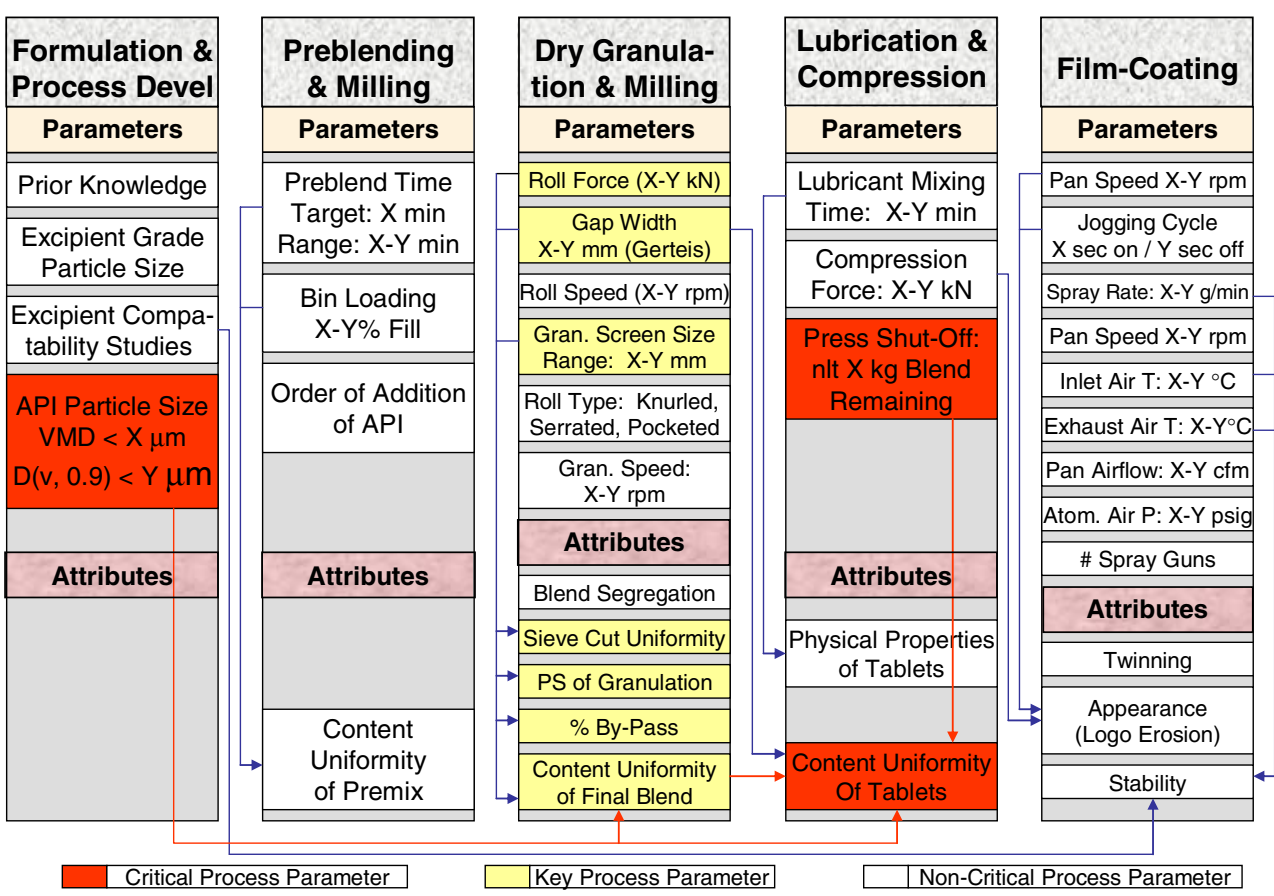

performed for the initial investigation into the importance of quality attributes and process parameters.

The quality of raw materials (including API, solvents, starting materials, excipients, and packaging components) should be assessed, and any critical quality attributes identified. As development continues, additional risk assessments can occur that define subsequent experiments that lead to an understanding of the interactions between different attributes and process parameters. In addition, multivariate models based on chemistry, biotechnology, or engineering fundamentals can be used to build the Design Space. These models can be based on first principles, be empirical in nature, or a combination of both. The intent of the experimentation and modeling is to create an understanding of all variables that impact CQAs, and represent the linkage in the form of a Design Space. This representation includes key links to risk assessment, the Control Strategy, and the Pharmaceutical Quality System.

The Design Space is linked to criticality through the results of risk assessment, which determines the associated CQAs and CPPs. It describes the multivariate functional relationships between CQAs and the CPPs that impact them, and should include their linkage to or across unit operations. Such relationships are arrived at by iterative application of risk assessment and experimental design, modeling, as well as the use of literature and prior experience.

The Design Space also contains the proven acceptable ranges $(\mathrm{PAR})^{7}$ for CPPs and acceptable values for their

\footnotetext{
${ }^{7}$ The proven acceptable range (PAR) is a characterized range of a process parameter for which operation within this range, while keeping other parameters constant, will result in producing a material meeting relevant quality criteria [4].
}

associated CQAs. Normal operating ranges are a subset of the Design Space and are managed under the company's Pharmaceutical Quality System. The Design Space may also contain operating ranges for process parameters classified in the intermediate criticality category discussed previously. Information regarding site and scale of manufacture may also be included, depending on the quality of the process knowledge upon which the Design Space is based.

Further details may be found in the paper by the PQLI Design Space Task Team: PQLI Design Space [19] in this Volume of the Journal of Pharmaceutical Innovation.

\section{Control Strategy}

\section{ICH Q10 defines a control strategy as}

"a planned set of controls derived from current product and process understanding that assures process performance and product quality. The controls can include parameters and attributes related to drug substance and drug product materials and components, facility and equipment operating conditions, in process controls, finished product specifications and the associated methods and frequency of monitoring and control.”

Control Strategy is not a new concept - products have always had a more or less explicit control strategy - but in ICH Q8(R) (Step 2) document a 'Minimal Approach' to Control Strategy is contrasted with the 'Enhanced, Quality by Design Approach'. In the latter, the control strategy is closely linked to both criticality and the Design Space. 
Fig. 5 Drug product design space for an oral solid dosage form

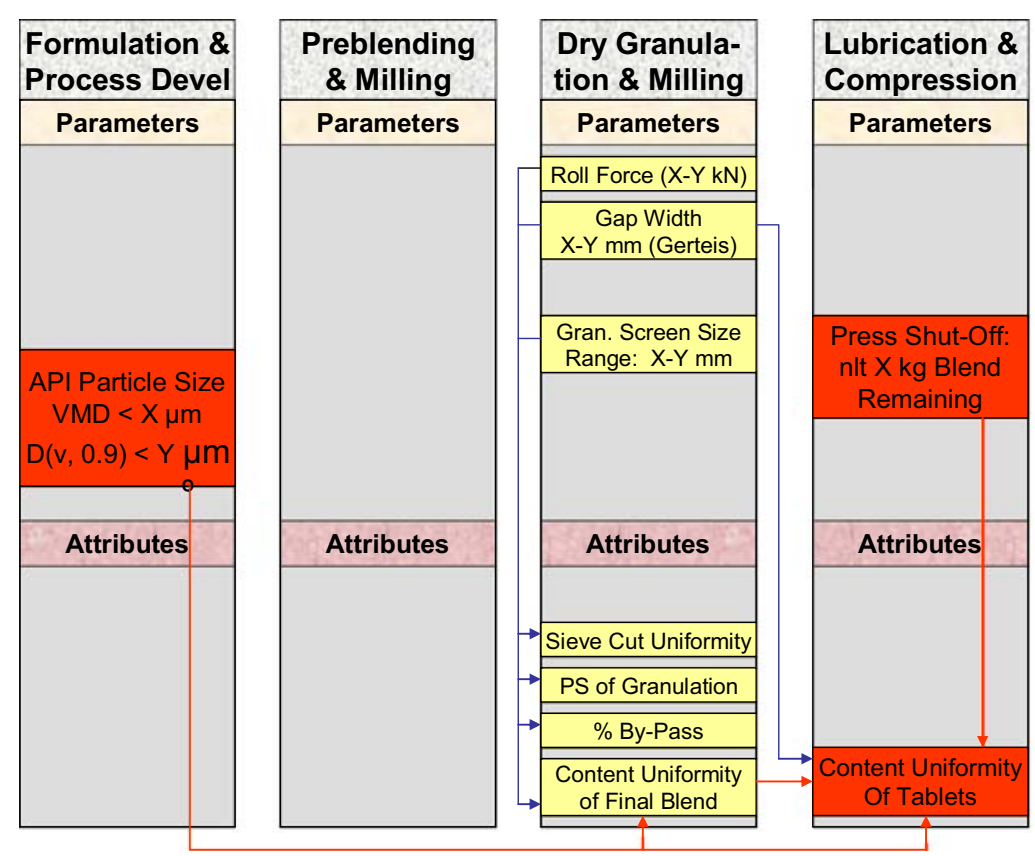

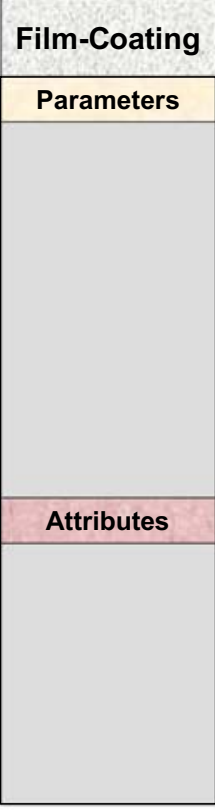

The results of the risk assessment identify those CQAs and CPPs that are included in the Design Space and subsequently must be included in the Control Strategy. The Control Strategy may include, for example, raw material purchase specifications, API characteristics, operating ranges for process parameters, in-process controls and their corresponding acceptance criteria, release testing, and API or drug product specifications and their acceptance criteria.

The ISPE PQLI Control Strategy Task Team has proposed a model that is intended primarily as a tool for pharmaceutical companies to facilitate communication and understanding of the concept and provide a framework for a structured approach to the development and implementation of a Control Strategy, particularly using the 'enhanced approach' described in ICH Q8R. As shown in Fig. 3, this model contains three levels showing links from the finished product CQAs and other objectives through the manufacturing operations to the controls by which these are achieved. Two columns distinguish between patient and business requirements.

At Level 1 the Critical Quality Attributes (CQAs) and other requirements are identified. Level 2 considers the critical process parameters, material attributes and compo-
Fig. 6 Drug product design space with control strategy for an oral solid dosage form

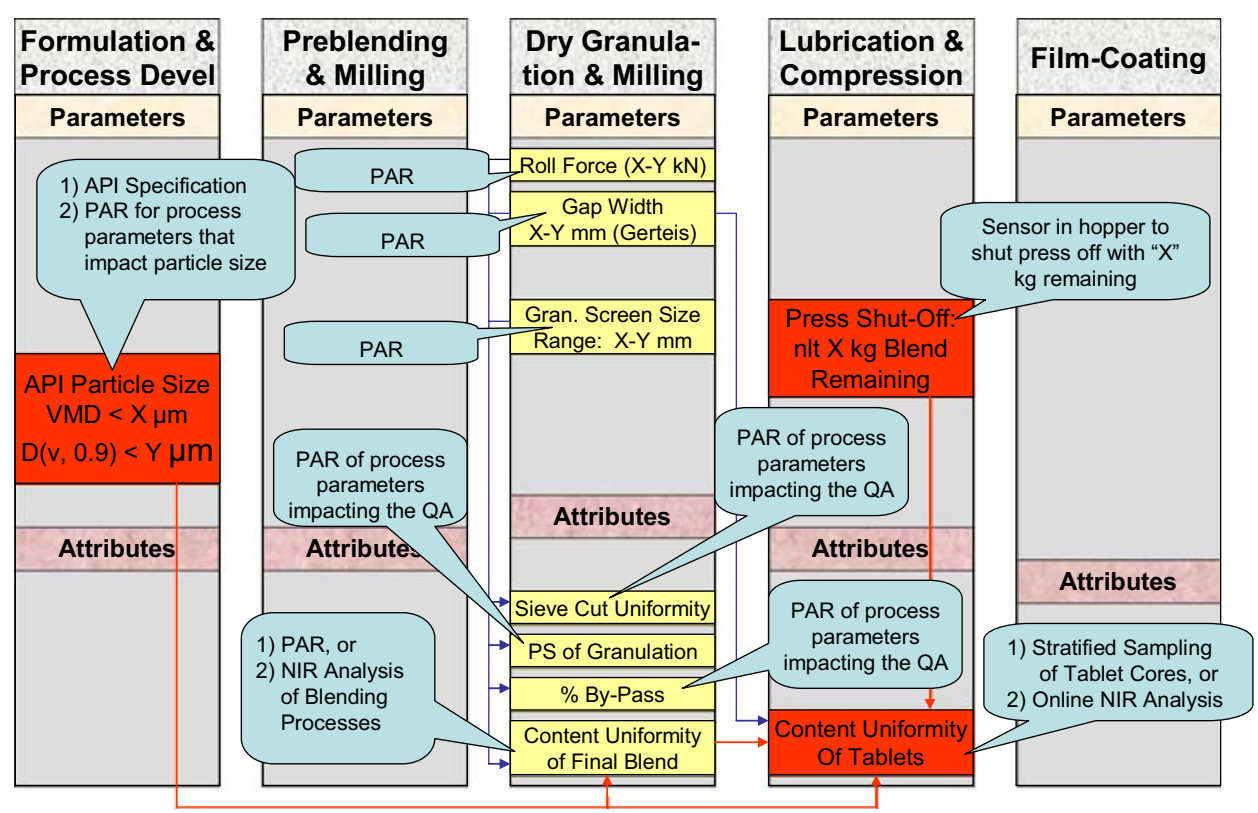

PAR $=$ Proven Acceptable Range for a process parameter . 
nents involved in meeting the CQA requirements. Level 3 covers the actual analytical, automation, and other controls of the Level 2 identified parameters and attributes.

This Control Strategy Model is described in more detail in the paper PQLI Control Strategy Model and Concepts [20] in this Volume of the Journal of Pharmaceutical Innovation.

\section{Proposed Approach for the Presentation of Design Space and Control Strategy}

The presentation of the Design Space may start with a brief overview explaining the process that was used during development to assess criticality, define the Design Space, and identify acceptable Control Strategies. This may include

- Definitions of terms used, especially any that have not been previously published or differ from those defined in ICH guidance documents.

- The risk assessment process, including iteration where appropriate.

- The identification of CQAs, links to clinical and toxicity studies for safety and efficacy, and their functional relationships with both process parameters and other quality attributes

- How prior knowledge, experience, or scientific literature were used during the initial risk assessments

- The link between risk assessments and the generation of experimental plans (e.g. based on DOE) and/or models to identify the CPPs and their acceptable operating ranges.

- How the development process evolved to obtain additional knowledge and information to obtain further clarity of the criticality of quality attributes and process parameters through process optimization.

- Information to assist the reader in the interpretation of the Design Space.

Figure 4 gives an example of how to present the Knowledge Space for an oral solid dosage form. This presents a pictorial summary of process knowledge and understanding. It combines columns containing summaries of the scientific information obtained for each unit operation into a single diagram, and links information across the entire manufacturing process.

Figure 5 shows how the Design Space, which is derived from the information contained in the Knowledge Space, may be presented. CQAs and CPPs are part of Design Space, but those attributes and parameters that only impact business drivers (such as processing times or cost) are not included. The Design Space is, therefore, derived from the knowledge space by removing non-critical quality attributes and process parameters that do not impact the safety, efficacy, or quality of the product. The Design Space may also contain those quality attributes or process parameters characterized in the intermediate ("X") category contained in the criticality decision in Fig. 2, if applicable. The linkage between CPPs and CQAs, including the impact of upstream and downstream processes, is also presented. Functional relationships may be shown to provide an understanding of all variables that impact CQAs.

Figure 6 links the Control Strategy to the Design Space. Appropriate controls should be implemented for CQAs and CPPs to ensure that product of acceptable quality is produced. Figure 6 illustrates where various elements of control are incorporated into the process (e.g., specifications, proven acceptable ranges for process parameters, PAT, and engineering controls).

The QbD principles discussed should be incorporated during the preparation of the CTD to produce a sciencebased submission. The manufacturing description should be an extension of the Design Space and include proven acceptable ranges (PAR) for CPPs. In some instances, the entire Design Space may be included as part of the manufacturing description. Information for non-critical quality attributes and process parameters should be omitted from the manufacturing description, as they do not impact the safety, efficacy, or quality of the product.

\section{References}

1. Product quality lifecycle implementation: practical approach to QbD, ISPE Washington Conference, Arlington, VA, 6-7 June, 2007.

2. Product Quality Lifecycle Implementation (PQLI): Practical approach to quality by design, ISPE 2007 Berlin Conference, Berlin, Germany, 19-September-2007.

3. International conference on harmonisation of technical requirements for registration of pharmaceuticals for human use, ICH Harmonised Tripartite Guideline, Pharmaceutical Development Q8, Step 4, 10-November-2005.

4. International Conference on Harmonisation, ICH Draft: Step 1, Q8 (R1) Pharmaceutical development revision 1, Step 3, November 2007.

5. International Conference on harmonisation of technical requirements for registration of pharmaceuticals for human use, ICH harmonised tripartite guideline, quality risk management Q9, Step 4, 9-November-2005.

6. International Conference on harmonisation of technical requirements for registration of pharmaceuticals for human use, ICH harmonised tripartite guideline, pharmaceutical quality system Q10, Step 2, 9-May-2007.

7. Draft PQLI summary update report, Version V04, product quality lifecycle implementation (PQLI), ISPE, 14-September-2007, http://www.ispe.org/cs/pqli_product_quality_lifeycle_implementation/draft pqli summary update_report.

8. E-RC3a - Product Quality Lifecycle Implementation (PQLI): a practical approach to QbD, 2007 ISPE Annual Meeting, 5November-2007, Las Vegas, Nevada.

9. E-RC3b - PQLI design qualification and design review (Continued), 2007 ISPE Annual Meeting, 6-November-2007, Las Vegas, Nevada. 
10. Janet Woodcock, pharmaceutical quality in the 21 st century - an integrated systems approach, AAPS workshop: pharmaceutical quality assessment - a science and risk-based CMC approach in the 21st Century, North Bethesda, MD, 5-October-2005.

11. Procedures for performing a failure mode, effects and criticality analysis, United States Military Procedure MIL-P-1629, 9-November-1949.

12. Pharmaceutical CGMPs for the 21st century: a risk-based approach; a science and risk-based approach to product quality regulation incorporating an integrated quality systems approach, United States Department of Health and Human Services, U.S. Food and Drug Administration, 21-August-2002 (http://www.fda. gov/oc/guidance/gmp.html).

13. PAT - A framework for innovative pharmaceutical development, manufacturing, and quality assurance, United States Department of Health and Human Services, U.S. Food and Drug Administration, Center for Drug Evaluation and Research (CDER), Center for Veterinary Medicine (CVM), Office of Regulatory Affairs (ORA), Pharmaceutical CGMPs, September 2004.

14. EMEA PAT Team Position Paper, http://www.emea.europa.eu/ Inspections/docs/PATGuidance.pdf
15. International conference on harmonisation of technical requirements for registration of pharmaceuticals for human use, ICH harmonised tripartite guideline, specifications: test procedures and acceptance criteria for new drug substances and new drug products: chemical substances Q6A, Step 4, 6-October-1999.

16. ISPE Conference on Innovation, Copenhagen, Denmark, April $7-$ 11,2008

17. Reported at ICH meeting "Step 4 for the ICH6 Program," Brussels, 17-18 July, 2003.

18. Criticality in PQLI. DOI 10.1007/s12247-008-9033-3.

19. PQLI Design Space. DOI 10.1007/s12247-008-9034-2.

20. PQLI Control Strategy Model and Concepts. DOI 10.1007/ s12247-008-9035-1.

If you would like to provide comments to the authors regarding this paper, email feedback@ispe.org and include "PQLI Key Topics" in the subject line. 